\title{
Natural History of Allergy to Hen's Egg: A Prospective Study in Children Aged 6 to 12 Years
}

\author{
Hiroaki Taniguchia, b Kiyotake Ogura ${ }^{a}$ Sakura Satoc, d Motohiro Ebisawac, d \\ Noriyuki Yanagida ${ }^{a}$ \\ aDepartment of Pediatrics, National Hospital Organization Sagamihara National Hospital, Sagamihara, Japan; \\ ${ }^{b}$ Department of Pediatrics, Konan Medical Center, Kobe, Japan; 'Department of Allergy, Clinical Research Center \\ for Allergy and Rheumatology, National Hospital Organization Sagamihara National Hospital, Sagamihara, Japan; \\ ${ }^{\mathrm{d} C}$ Course of Allergy and Clinical Immunology, Juntendo University Graduate School of Medicine, Tokyo, Japan
}

\section{Keywords}

Anaphylaxis · Food hypersensitivity $\cdot$ Hen's egg $\cdot$ Natural history · Tolerance

\begin{abstract}
Introduction: There are limited reports on the natural history of hen's egg (HE) allergy (HEA) in children $<6$ years. We aimed to investigate the natural history of HEA in children aged 6-12 years and the factors affecting its tolerance acquisition. Methods: Using the database in our hospital, a total of 137 patients diagnosed with a definitive immediate-type reaction to HE when they turned 6 years were enrolled, and the natural course of HEA was prospectively examined until patients turned 12 years. Tolerance was defined as being able to pass an oral food challenge to consume a half or whole heated HE or consume heated HE freely without symptoms. Thirty patients $(21.9 \%)$ who were enrolled for oral immunotherapy and 21 (15.3\%) who discontinued follow-up were considered dropouts. Kaplan-Meier estimation was used to evaluate the rate of tolerance. Results: Fifty-five of the 137 patients (40.1\%) had a previous HE anaphylaxis history; 61 (44.5\%) patients had acquired tolerance to HE by age 12 years; and 25 (18.2\%) continued total or partial HE
\end{abstract}

elimination. The estimated acquired tolerance rates by ages 7,9 , and 12 years were $14.6 \%, 40.8 \%$, and $60.5 \%$, respectively. A previous history of $\mathrm{HE}$ anaphylaxis before 6 years of age, reacting to small amounts of heated $\mathrm{HE}$ by 6 years of age, and higher ovomucoid-specific immunoglobulin $E$ values at the same age were associated with persistent HEA. Conclusion: This study provides important insights into the natural course of HEA beyond early childhood, with the acquisition of $\mathrm{HE}$ tolerance continuing throughout the duration of the study.

(c) 2021 S. Karger AG, Basel

\section{Introduction}

Food allergy has become an increasingly prominent condition worldwide; it results in allergic reactions of varying severity and is a burden to patients and their families. The prevalence of food allergy in Japan is estimated at around 5-10\% among infants [1] and 4.5\% among school-age children [2]. Food sensitization in the early

Edited by: O. Palomares, Madrid. karger@karger.com www.karger.com/iaa
(C) 2021 S. Karger AG, Basel

Karger"
Correspondence to:

Noriyuki Yanagida, sagami@ foodallergy.jp 
years of life is related to other allergic diseases, including asthma and allergic rhinitis [3].

Hen's egg (HE) is one of the most common causes of food allergy around the world, with a reported prevalence of $1.6-10.1 \%[4,5]$. A previous Japanese study focusing on immediate-type food allergy found that $\mathrm{HE}$ was the most commonly observed causative food allergen, accounting for $39.0 \%$ of all such reactions across all agegroups [6].

The natural history of HE allergy (HEA) has been previously reported in various countries [7-15]. The rate of tolerance varies across reports, from $12 \%$ to $73 \%$ by 6 years of age. Some, but not all, of the participants of those studies received oral food challenge (OFC) either for HEA diagnosis or for the determination of whether tolerance to $\mathrm{HE}$ was acquired. Our facility has previously reported on the natural history of HEA in children aged 0-3 years [14] and 0-6 years [15] in Japan. In those reports, the proportions of patients who had acquired tolerance to HEA were $30 \%$ and $73 \%$ at ages 3 and 6 years, respectively. Few studies however have focused on the natural history of HEA beyond the age of 6 years. Savage et al. [9] reported the rate of tolerance in the US as $37 \%$ by 10 years of age and $68 \%$ by 16 years of age. In a longitudinal study of HEA resolution conducted by Clark et al. [10], the estimated rate of tolerance to well-cooked $\mathrm{HE}$ was $60 \%$ by the age of 6 years and $94 \%$ by the age of 12 years and 2 months.

To the best of our knowledge, the natural history of HEA beyond the age of 6 years has not been previously reported in Japan. Such a study could provide important insights into the natural history of HEA and useful information on HEA management in patients with persistent HEA until school age. Therefore, we determined the natural history and factors influencing the acquisition of tolerance among HEA patients aged 6-12 years.

\section{Materials and Methods}

\section{Database}

The iAnet is an internal comprehensive food allergy patient database (UMIN000013561) designed with the cooperation of Hitachi Solutions Create (Tokyo, Japan); it contains patient data including sex, date of birth, birth weight, gestational age at birth, type of delivery (normal vaginal delivery or cesarean section), feeding patterns during infancy (exclusive or partial breastfeeding or exclusive formula feeding), descriptions of food allergy, comorbid allergic diseases, and familial allergic history. In our hospital, patient data are usually obtained at the first visit. Comorbid allergic diseases, if present, were diagnosed by the attending pediatric allergists. The descriptions of food allergy included the names of causative foods, time of diagnosis, reason for the elimination of the causative food, history of anaphylaxis related to individual causative foods, and current status of food allergy (acquired tolerance, partial, or total elimination). Individual data are updated (to reflect the changes in the patients' allergic disease status) prospectively once a year.

\section{Participants}

We enrolled children with food allergies who visited the National Hospital Organization, Sagamihara National Hospital, and who were registered on iAnet. For this study, patient data matching the set inclusion criteria were extracted from iAnet. Inclusion criteria were date of birth between January 2002 and December 2003; HEA diagnosed with the use of either a positive OFC or physician-diagnosed immediate-type reaction to HE; having visited the National Hospital Organization, Sagamihara National Hospital, before age 6 years; and had not acquired tolerance to HEA by this age (shown in Fig. 1). Enrollment at age 6 years was chosen as a sequel to a previous retrospective study on HEA, in which the natural history of HEA was investigated from age 0 to 6 years at our facility [15]. Those whose HEA was not confirmed through an OFC or a physician-diagnosed immediate-type reaction, those who visited our facility after they turned 6 years of age, and those who had already acquired tolerance to HE before 6 years of age were excluded.

\section{Follow-Up}

We prospectively studied the natural course of HEA from age 6 years until the patients turned 12 years. The follow-up age (6-12 years) was chosen to match the age span of elementary education in Japan.

During the follow-up period, the patients underwent periodical OFCs, usually annually. If the OFC was confirmed as being negative and patients were able to consume certain amounts of HE (i.e., heated egg yolk), they were advised to consume up to that same amount until the next OFC. If the OFC was positive, patients were instructed to continue with total elimination or to consume the amount that was available prior to the positive OFC $[16,17]$. Blood tests to measure IgE values were usually performed once a year or at the attending pediatrician's request. Specific IgE values were measured using ImmunoCAP ${ }^{\mathrm{TM}}$ (Thermo Fisher Scientific/Phadia, Uppsala, Sweden).

\section{End Point}

The primary end point was the rate of HE tolerance acquisition by 12 years of age. Tolerance was defined as being able to pass the OFC to a half or whole heated HE and/or consume HE freely without symptoms (tolerance group). Patients who passed the OFC were instructed to consume $\mathrm{HE}$ at home to confirm their tolerance. The times to the acquisition of tolerance were recorded for patients who acquired tolerance to HE. Those with complete or partial HE elimination by the age of 12 years were considered as having persistent HEA (persistent HEA group). Patients who discontinued the follow-up and those who enrolled for oral immunotherapy (OIT) for HE were considered as dropouts.

The secondary end points were the patients' characteristics at the age of 6 years according to the tolerance and elimination groups. These characteristics were patients' sex, body weight at birth, gestational age at birth, type of delivery, rate of exclusive breastfeeding, history of anaphylaxis due to HE before the age of 6 
Fig. 1. Patient enrollment and clinical outcomes. In total, 137 patients were enrolled in this prospective study. Sixty-one patients (45\%) acquired tolerance, while 25 (18\%) continued elimination. HEA, hen's egg allergy; OIT, oral immunotherapy.

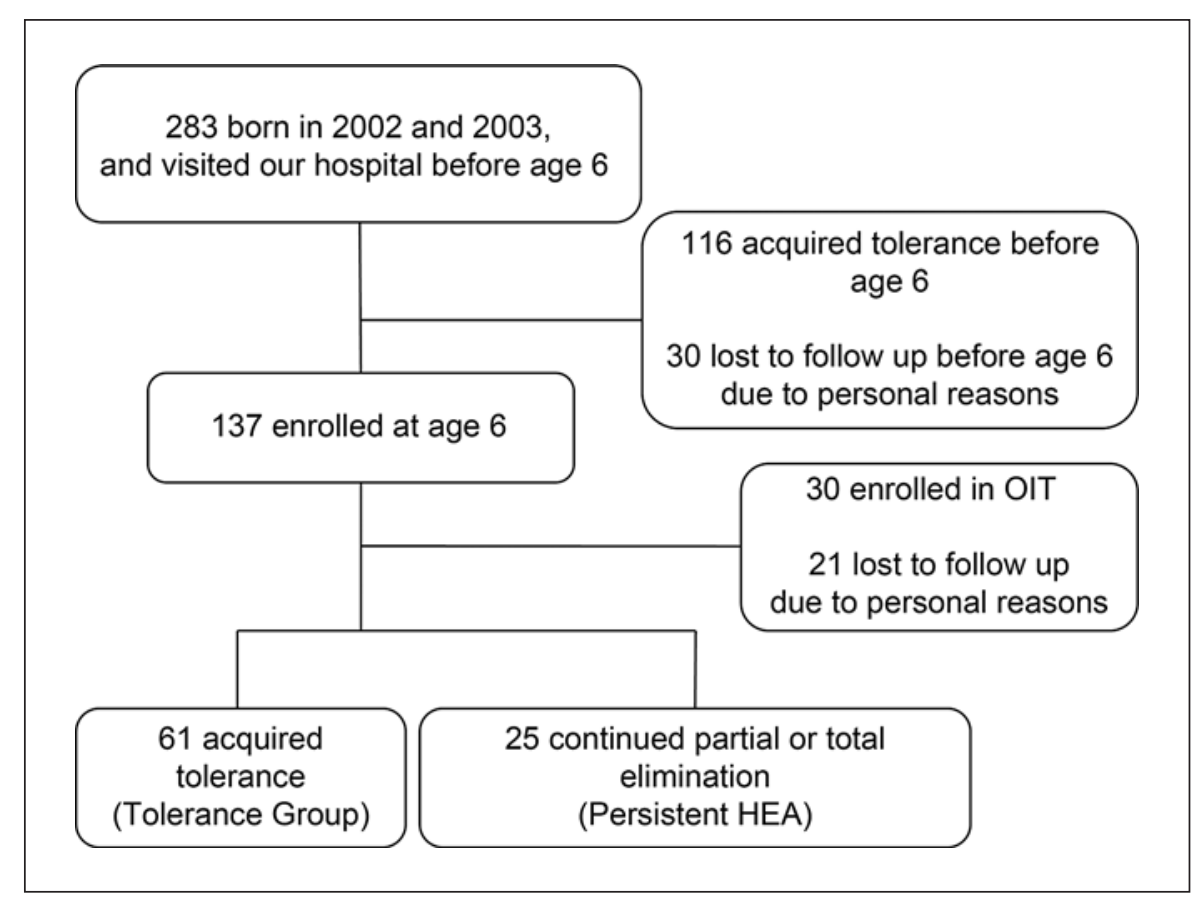

years, the number of food allergens other than HE, comorbid allergic diseases at the age of 6 years (atopic dermatitis, bronchial asthma, allergic rhinitis, and allergic conjunctivitis), total IgE val$\mathrm{ue}$, and the specific IgE values of egg white (EW) and ovomucoid (OVM).

\section{Statistical Analyses}

To depict the HEA resolution trend from 6 to 12 years of age, Kaplan-Meier survival estimation curves were generated. For the comparison of the patients' characteristics and allergen-specific IgE values between the tolerance and persistent HEA groups, we applied Fisher's exact test for categorical variables and the MannWhitney $\mathrm{U}$ test for continuous variables. The hazard ratio and $95 \%$ confidence interval comparing patients with the factors influencing persistent HEA were reported based on a multivariate Cox proportional hazards model. The covariates included in the Cox proportional hazards model were selected from among factors that were significantly associated with persistent HEA in the previous reports or were factors whose $p$ values were $<0.10$ in the comparison of patients' characteristics.

As an additional evaluation, data on those who participated in OIT and those lost to follow-up were analyzed in 2 additional ways. The first approach was more stringent and designated them as having continued with HE elimination until they turned 12 years of age. In the second approach, we focused on the OIT results, and those who acquired sustained unresponsiveness to HE through OIT were also counted as having acquired tolerance at the time, at which sustained unresponsiveness to HE was achieved.

In the post hoc analyses, we compared the patients by dividing them based on whether their EW- and OVM-specific IgE values were above or below the median level. Kaplan-Meier survival estimation curves were generated, considering those who enrolled for OIT and those who were lost to follow-up as dropouts. Moreover,
Kaplan-Meier survival estimation curves were generated comparing the patients based on factors that were significant in the Cox proportional hazards model. Additionally, we generated KaplanMeier survival estimation curves combining 2 factors that were significant in the Cox proportional hazard model. The KaplanMeier curves of the 2 groups were compared using a log-rank test. $p$ values $<0.05$ were considered statistically significant. All statistical analyses were performed using $\mathrm{R}$ version 3.3.2 (The R Foundation for Statistical Computing, Vienna, Austria).

\section{Ethical Considerations}

The patients or their guardians provided oral or written consent at the time of enrollment in iAnet. Those who chose not to be enrolled were excluded from this study. The board of ethics at Sagamihara National Hospital reviewed and approved iAnet (Rinri 2007-Oct.-009).

\section{Results}

\section{Patient Enrollment and Outcomes}

As shown in Figure 1, a total of 137 patients (95 boys and 42 girls) who were registered in iAnet before turning 6 years of age were enrolled in the study at the point in which they turned 6 years. By the time they turned 12 years, 61 patients $(44.5 \%)$ had acquired tolerance (tolerance group), while 25 (18.2\%) continued with the total or partial elimination of $\mathrm{HE}$ (persistent HEA group). HE tolerance was confirmed by the use of a heated $\mathrm{HE} \mathrm{OFC} \mathrm{in}$ $46(75.4 \%)$ of the 61 patients, whereas in 15 (24.6\%) pa-
16

Int Arch Allergy Immunol 2022;183:14-24 DOI: $10.1159 / 000518522$
Taniguchi/Ogura/Sato/Ebisawa/Yanagida 
Fig. 2. Kaplan-Meier estimation curve of HEA resolution from 6 to 12 years of age. Shaded areas indicate 95\% CIs. HEA, hen's egg allergy; CIs, confidence intervals.

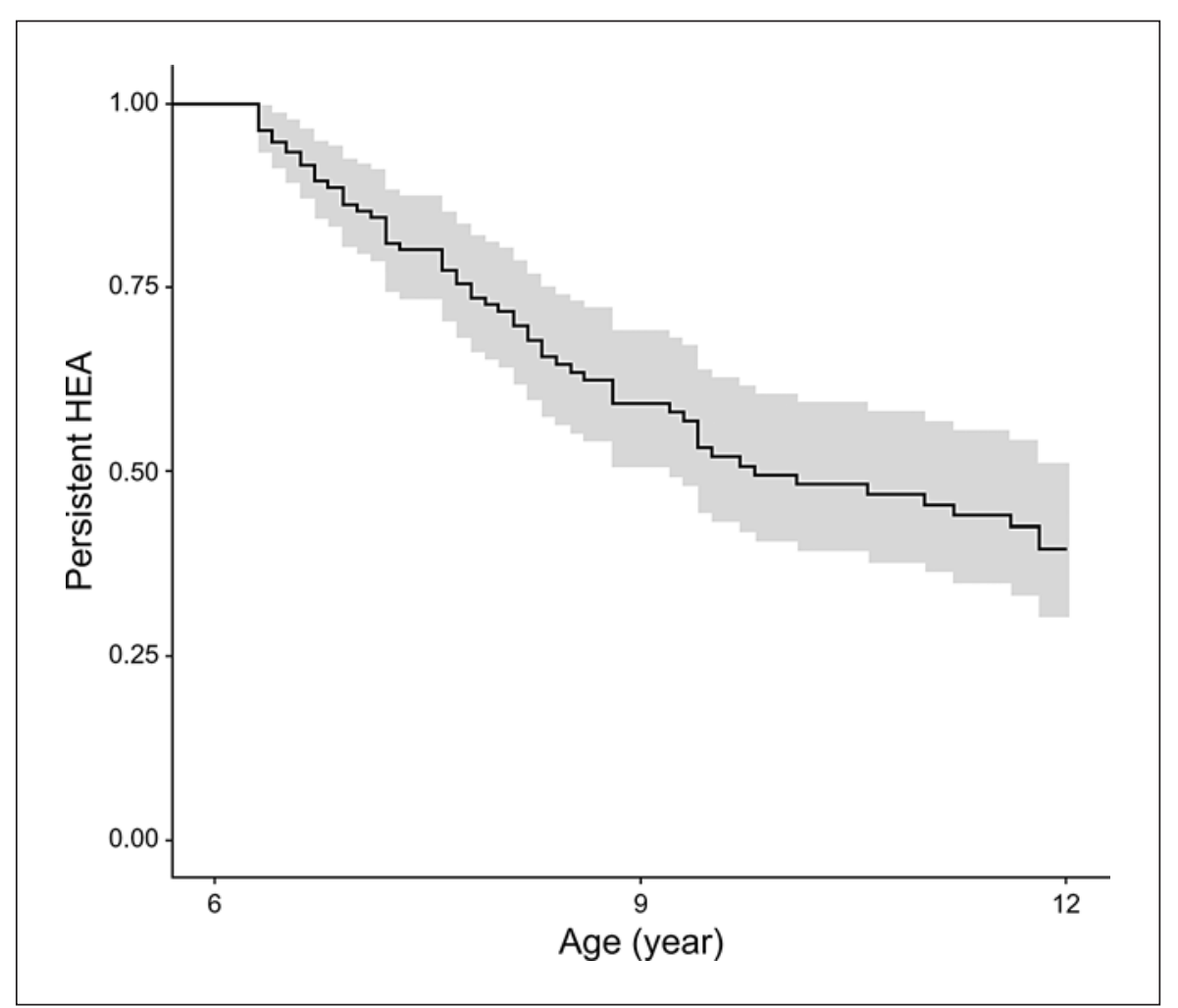

tients, tolerance was confirmed when HE was introduced at home or through accidental ingestion. Thirty (21.9\%) patients were enrolled for OIT (OIT group), while 21 (15.3\%) discontinued follow-up during the study period. Of those in the OIT group, 18 (60.0\%) achieved sustained unresponsiveness, $9(30.0 \%)$ continued with OIT at age 12 years, and $3(10.0 \%)$ dropped out of OIT.

\section{Estimated Rate of HEA Resolution}

Using Kaplan-Meier survival estimation, the estimated percentage of HEA resolution was calculated (shown in Fig. 2). When we designated the patients in the OIT group and those who were lost to follow-up as dropouts, the percentages of resolution by age 7,9 , and 12 years were $14.6 \%, 40.8 \%$, and $60.5 \%$, respectively. The median age at HEA resolution was 9.8 years.

Further, we also estimated the HEA resolution rates using different approaches. Online suppl. Figure 1 (see www.karger.com/doi/10.1159/000518522 for all online suppl. material) shows the Kaplan-Meier survival estimation of HEA resolution when the OIT group and those lost to follow-up are designated as "continued HE elimination until age 12 years." Here, the percentages of resolution by 7,9 , and 12 years of age were $13.9 \%, 33.6 \%$, and
$44.5 \%$, respectively. Online suppl. Figure 2 shows the results when the OIT results are factored in. Those who achieved sustained unresponsiveness to HE through OIT were also counted as having acquired tolerance at the time at which they achieved sustained unresponsiveness to HE. Those who were lost to follow-up were considered as dropouts. Here, the percentages of resolution by 7, 9, and 12 years of age were $15.0 \%, 43.8 \%$, and $65.2 \%$, respectively. The median age at HEA resolution was 9.7 years.

\section{Patient Characteristics}

Table 1 shows the background characteristics of all the patients at the age of 6 years. All the patients had immediate-type HEA, with 55 patients (40.1\%) experiencing HE anaphylaxis. The patients were also highly atopic, with 130 (94.8\%) showing comorbid atopic dermatitis at the age of 6 years.

\section{Comparisons of the Patient Characteristics}

The background characteristics of those in the tolerance and persistent HEA groups are presented in Table 2. Between the 2 groups, a statistically significant difference was observed only in the number of other food allergens at the age of 6 years and the number of patients who passed 
Table 1. Background characteristics of all patients

\begin{tabular}{lc}
\hline & $\begin{array}{c}\text { All patients } \\
(n=137)\end{array}$ \\
\hline Male & $95(69.3)$ \\
Term delivery & $131(95.6)$ \\
Caesarian section & $12(8.8)$ \\
Total breastfeeding & $31(22.6)$ \\
Secondhand smoking & $56(49.9)$ \\
Parental history of allergic diseases ${ }^{\dagger}$ & $123(89.8)$ \\
Age of the first visit, years & $2.3(1.0-4.5)$ \\
Age of the first OFC, years & $5.5(3.9-6.3)$ \\
Food allergens at the age of 6 years, $n$ & $2(1-4)$ \\
Comorbid diseases & $86(62.8)$ \\
$\quad$ Bronchial asthma & $130(94.9)$ \\
$\quad$ Atopic dermatitis & $27(19.7)$ \\
$\quad$ Allergic rhinitis & $15(10.9)$ \\
Allergic conjunctivitis & $55(40.1)$ \\
History of anaphylaxis to HE & \\
$\quad$ of 6 years & $46(33.6)$ \\
Total IgE value, IU/mL & $1,080(40-2,105)$ \\
EW-specific IgE value, $\mathrm{kU} \mathrm{A}_{\mathrm{A}} / \mathrm{L}$ & $24.4(7.2-41.5)$ \\
OVM-specific IgE value, kU $/ \mathrm{L}$ & $12.6(4.8-33.3)$ \\
\hline
\end{tabular}

Categorical variables are presented as $n(\%)$, and continuous variables as median (interquartile range). OFC, oral food challenge; HE, hen's egg; EW, egg white; OVM, ovomucoid; IgE, immunoglobulin E. ${ }^{\dagger}$ One or both parents have at least one of the following: food allergy, bronchial asthma, atopic dermatitis, allergic rhinitis, or allergic conjunctivitis.

a heated egg yolk OFC by this age. Other factors, including a history of $\mathrm{HE}$ anaphylaxis were not significant. The results of the comparisons of the background characteristics of those in the OIT group with those in the tolerance and persistent HEA groups are shown in online suppl. Table 1. The OIT group had significantly higher rates of HE anaphylaxis experience compared to the tolerance group ( $63 \%$ vs. $26 \%, p=0.002$ ). There were no significant differences between the OIT and persistent HEA groups in any of the background characteristics examined.

The results of the multivariate Cox hazard model analysis are presented in Table 3. A history of HE anaphylaxis, a positive heated egg yolk OFC before the age of 6 years, and higher OVM-specific IgE values at the age of 6 years were significantly associated with persistent HEA. The proportional hazards assumption was verified graphically and statistically (data not shown).

\section{Specific IgE Values and Tolerance}

The EW- and OVM-specific IgE values $\left(\mathrm{kU}_{\mathrm{A}} / \mathrm{L}\right)$ at the age of 6 years were significantly lower in the tolerance group than in the persistent HEA group (median 12.5 vs. $39.8, p=0.002$; and 7.4 vs. $33.2, p=0.0003$, respectively). Figure 3 shows the yearly changes in the specific IgE values. The yearly EW-specific IgE values were significantly lower in the tolerance group until 7 years of age. Although the tolerance group had relatively lower values than the persistent HEA group after 8 years of age, significance was not observed. A similar significance level was observed in the OVM-specific IgE values until 9 years of age. The OIT group also had significantly higher EW- and OVM-specific IgE values than those of the tolerance group (median 29.1 vs. $12.5, p=0.025$; and 18.1 vs. 7.4 , $p=0.002$, respectively).

\section{Patient Characteristics, Specific IgE Values, and Tolerance}

A comparison of patients with specific IgE values above and below the overall median level $\left(24.4 \mathrm{kU}_{\mathrm{A}} / \mathrm{L}\right.$ for $\mathrm{EW}$ - and $12.5 \mathrm{kU}_{\mathrm{A}} / \mathrm{L}$ for OVM-specific IgE) is shown in Figure $4 \mathrm{a}$ and $\mathrm{b}$. Using Kaplan-Meier survival estimation, among patients whose EW-specific IgE at the age of 6 years were $<24.4 \mathrm{kU}_{\mathrm{A}} / \mathrm{L}$, the estimated percentages of HEA resolution at 7,9 , and 12 years of age were $24.0 \%$, $49.5 \%$, and $74.8 \%$, respectively, and among patients whose $\mathrm{EW}$-specific IgE values were $\geq 24.4 \mathrm{kU}_{\mathrm{A}} / \mathrm{L}$, the rates were $6.3 \%, 32.8 \%$, and $50.2 \%$, respectively (shown in Fig. 4a). Among patients whose OVM-specific IgE at the age of 6 years when the IgE values were $<12.5 \mathrm{kU}_{\mathrm{A}} / \mathrm{L}$ the rates of HEA resolution at 7, 9, and 12 years of age were $25.6 \%, 53.9 \%$, and $77.7 \%$,respectively, and among patients whose OVM-specific IgE values were $\geq 12.5 \mathrm{kU}_{\mathrm{A}} / \mathrm{L}$, the rates were $3.3 \%, 28.0 \%$, and $48.2 \%$, respectively (shown in Fig. 4b). In the log-rank test, those with IgE values below the median level were significantly more likely to acquire tolerance ( $p=0.014$ for EW and $p=0.003$ for OVM) than those with values above the median value.

The Kaplan-Meier survival estimation curves comparing patients with and without a history of HE anaphylaxis are shown in Figure 5a. In the log-rank test, those without a history of $\mathrm{HE}$ anaphylaxis were significantly more likely to acquire tolerance $(p=0.015)$.

The Kaplan-Meier survival estimation curves comparing patients who had and had not passed the heated egg yolk OFC by 6 years of age are shown in Figure 5b. In the log-rank test, those who had passed the heated egg yolk OFC by 6 years of age were significantly more likely to acquire tolerance $(p<0.001)$.

We also generated Kaplan-Meier survival estimation curves combining history of $\mathrm{HE}$ anaphylaxis, tolerance to heated egg yolk by the age of 6 years, and whether the
18

Int Arch Allergy Immunol 2022;183:14-24 DOI: $10.1159 / 000518522$
Taniguchi/Ogura/Sato/Ebisawa/Yanagida 
Table 2. Background characteristics comparisons between tolerance versus persistent HEA groups

\begin{tabular}{lcrr}
\hline & $\begin{array}{c}\text { Tolerance group } \\
(n=61)\end{array}$ & $\begin{array}{l}\text { Persistent HEA } \\
(n=25)\end{array}$ & $p$ value \\
\hline Male & $42(69)$ & $17(68)$ & $>0.999$ \\
Term delivery & $59(97)$ & $23(92)$ & 0.289 \\
Caesarian section & $5(8)$ & $2(8)$ & $>0.999$ \\
Total breastfeeding & $11(18)$ & $6(24)$ & 0.552 \\
Secondhand smoking & $26(43)$ & $6(24)$ & 0.146 \\
Parental history of allergic diseases ${ }^{\dagger}$ & $56(92)$ & $21(84)$ & 0.438 \\
Age of the first visit, years & $2.6(1.3-4.6)$ & $1.9(0.9-4.3)$ & 0.472 \\
Age of the first OFC, years & $5.6(4.2-6.1)$ & $5.4(3.5-7.0)$ & 0.640 \\
Food allergens at the age of 6 years, $n$ & $2(1-4)$ & $3(2-5)$ & $0.048^{*}$ \\
Comorbid diseases & & $20(80)$ & 0.083 \\
$\quad$ Bronchial asthma & $36(59)$ & $24(96)$ & $>0.999$ \\
$\quad$ Atopic dermatitis & $57(93)$ & $6(24)$ & 0.353 \\
$\quad$ Allergic rhinitis & $9(15)$ & $4(16)$ & 0.438 \\
$\quad$ Allergic conjunctivitis & $5(8)$ & $10(40)$ & 0.301 \\
History of anaphylaxis to HE & $16(26)$ & $4(16)$ & $0.025^{*}$ \\
Passed heated egg yolk OFC by the age of 6 & $26(43)$ & $1,375(754-2,155)$ & 0.388 \\
Total IgE value, IU/mL & $1,069(328-2,033)$ & & \\
\hline
\end{tabular}

Categorical variables are presented as $n(\%)$ and tested using Fisher's exact test. Continuous variables are presented as median (IQR) and tested using Mann-Whitney's U test. HEA, hen's egg allergy; OFC, oral food challenge; HE, hen's egg; EW, egg white; OVM, ovomucoid; IQR, interquartile range; IgE, immunoglobulin E. ${ }^{*} p$ value $<0.05 .{ }^{\dagger}$ One or both parents having at least one of the following: food allergy, bronchial asthma, atopic dermatitis, allergic rhinitis, or allergic conjunctivitis.

Table 3. Cox regression analysis of factors contributing to persistent HEA

\begin{tabular}{|c|c|c|}
\hline & Adjusted HR (95\% CI) & $p$ value \\
\hline Food allergens at the age of 6 years (per increase of 1 ), $n$ & $1.07(0.92,1.24)$ & 0.409 \\
\hline Comorbid bronchial asthma & $1.71(0.98,2.99)$ & 0.058 \\
\hline History of anaphylaxis to HE & $2.50(1.31,4.76)$ & $0.005^{*}$ \\
\hline Passed heated egg yolk OFC by the age of 6 years & $0.29(0.16,0.53)$ & $<0.001^{*}$ \\
\hline OVM-specific IgE value at the age of 6 years (per 10 -fold increase) & $2.94(1.96,4.43)$ & $<0.001^{*}$ \\
\hline
\end{tabular}

Multivariate Cox regression analysis was performed using the factors presented in this table as covariates. HEA, hen's egg allergy; HR, hazard ratio; CI, confidence interval; HE, hen's egg; OFC, oral food challenge; IgE, immunoglobulin E; OVM, ovomucoid. ${ }^{*} p$ value $<0.05$.

OVM-specific IgE values were higher or $<12.5 \mathrm{kU}_{\mathrm{A}} / \mathrm{L}$. The estimation curves are shown in online suppl. Figure 3. Those who did not have a history of anaphylaxis to HE and could tolerate heated egg yolk were significantly more likely to acquire tolerance to HE compared to the other groups of patients $(p<0.005)$. Conversely, those who had a history of anaphylaxis to HE and whose OVMspecific IgE values were $\geq 12.5 \mathrm{kU}_{\mathrm{A}} / \mathrm{L}$ were more unlikely to acquire tolerance to $\mathrm{HE}$ by 12 years of age.

\section{Discussion/Conclusion}

Herein, we presented the natural history of HEA in children from ages 6 to 12 years. All the investigated patients had immediate-type HEA, which was either an OFC- or physician-diagnosed immediate-type reaction to HE. More than $60 \%$ of the patients with HEA were estimated to have acquired tolerance by age 12 years.

The natural history of HEA has been reported previously, including in Japan [7-15]. Many of those reports were based on the follow-up of patients during the first 


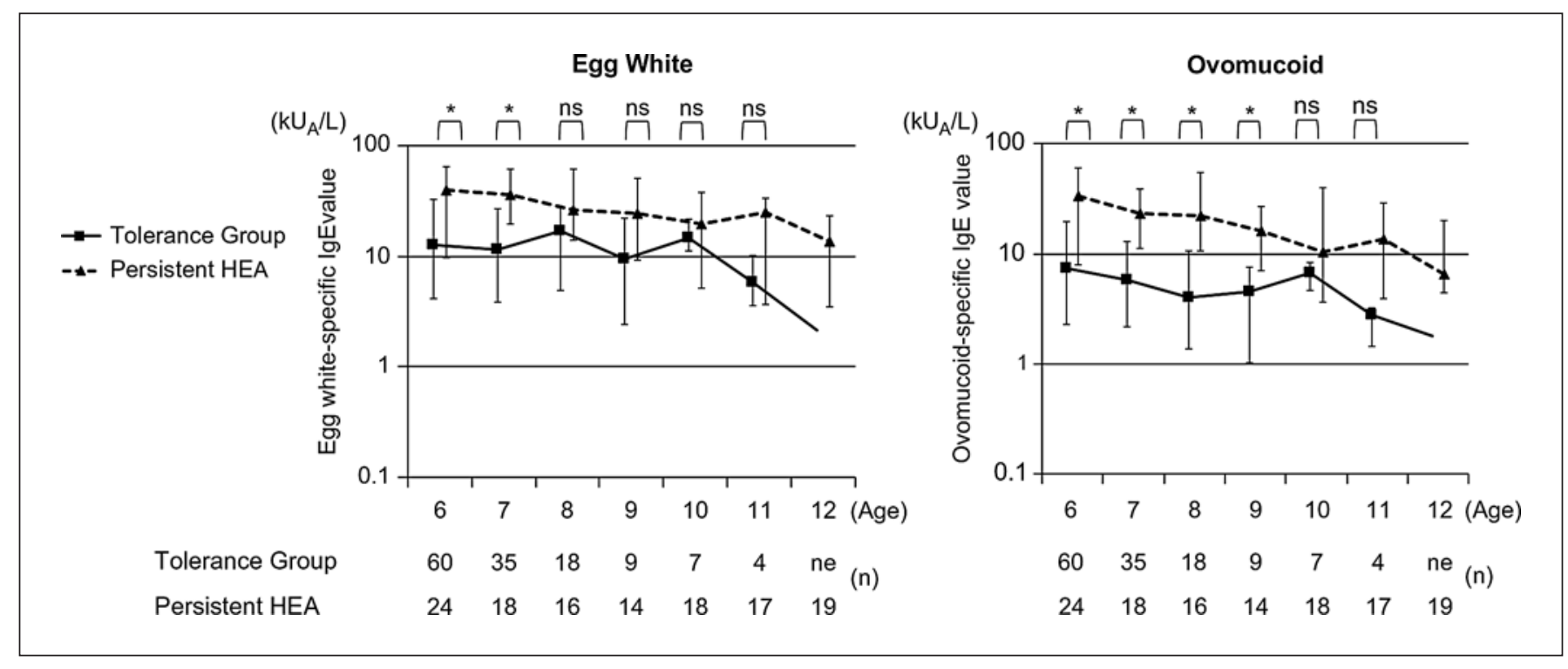

Fig. 3. Changes in the EW-specific IgE values (left) and OVM-specific IgE values (right). The triangle and square in the graph indicate median IgE values, while the upper and lower horizontal bars indicate the IQR. The number of patients who underwent IgE value evaluations is shown in the lower half of the figure. * $p$ value $<0.05$ calculated by a Mann-Whitney U test. ns, not significant; ne, not estimated; HEA, hen's egg allergy; IgE, immunoglobulin E; IQR, interquartile range; EW, egg white; OVM, ovomucoid.

few years of their lives. Few studies have focused on school-age children with HEA $[9,10]$. In the previous studies, some of the children who continued to have HEA beyond 6 years of age were able to achieve tolerance to $\mathrm{HE}$, although there were variations in the rates of achieving tolerance $[9,10]$. Our study results showed a similar trend, with $60.5 \%$ of the patients who still had HEA at the age of 6 years acquiring $\mathrm{HE}$ tolerance by the age of 12 years. Our results and those of the previous studies cannot be simply compared because our study enrolled patients at a starting age of 6 years, unlike the previous studies that investigated patients at much younger ages [7-15]. However, our study participants and their results may be better reflections of the real world. Additionally, our study participants underwent OFCs periodically, usually annually. This contributes to a more accurate assessment of the diagnosis of HE tolerance.

According to the previous reports, including 2 from our facility, the risk factors of HEA that persist beyond early childhood and into school age include the presence of comorbid bronchial asthma [15], atopic dermatitis [15], higher EW- and OVM-specific IgE values [11, 15], larger skin prick test wheals $[8,11]$, and systemic reactions or anaphylaxis on $\mathrm{HE}$ intake $[7,8,11,15]$. Some reports demonstrated that patients who tolerate baked eggs are more likely to be able to achieve HEA resolution $[12,18]$. Our study participants' background characteristics at 6 years of age showed similar characteristics, with relatively high EW- and OVM-specific IgE values and $40 \%$ of patients having experienced $\mathrm{HE}$ anaphylaxis. In addition, the proportion of patients who had already exhibited tolerance to smaller amounts of heated HE (heated egg yolk) was significantly higher in the tolerance than in the persistent HEA group, suggesting that those who can tolerate smaller $\mathrm{HE}$ amounts are more likely to achieve tolerance [16].

In a report by Savage et al. [9], the factors associated with a slower resolution of HEA in older age children were the presence of higher egg-specific IgE values, other atopic diseases, and other food allergens. In our study, multivariate Cox regression analysis revealed that a history of anaphylaxis to HE, not being able to pass a heated egg yolk OFC, and higher OVM-specific IgE values at 6 years of age were significantly associated with persistent HEA. However, other factors, including the presence of other atopic diseases, were not significantly associated with HEA resolution, unlike in the previous studies. This may also be due to the fact that, in our study, patients were followed up from 6 years of age. Therefore, the 
Fig. 4. Kaplan-Meier estimation curve of HEA resolution divided by the EW- and OVM-specific IgE values at 6 years of age. a Kaplan-Meier estimation curve of HEA resolution divided by the $\mathrm{EW}$-specific IgE values (EW) at 6 years of age. Black line indicates patients whose EW-specific IgE values at 6 years of age were $\geq 24.4 \mathrm{kU}_{\mathrm{A}} / \mathrm{L}$. Gray line indicates patients with values $<24.4 \mathrm{kU}_{\mathrm{A}} / \mathrm{L}$. Shaded areas indicate $95 \%$ CIs. b Kaplan-Meier estimation curve of HEA resolution divided by the OVM-specific IgE values at 6 years of age. Black line indicates patients whose OVM-specific IgE values at 6 years of age were $\geq 12.5 \mathrm{kUA} / \mathrm{L}$. Gray line indicates patients with values $<12.5 \mathrm{kUA} / \mathrm{L}$. Shaded areas indicate 95\% CIs. HEA, hen's egg allergy; EW, egg white; OVM, ovomucoid; CIs, confidence intervals.

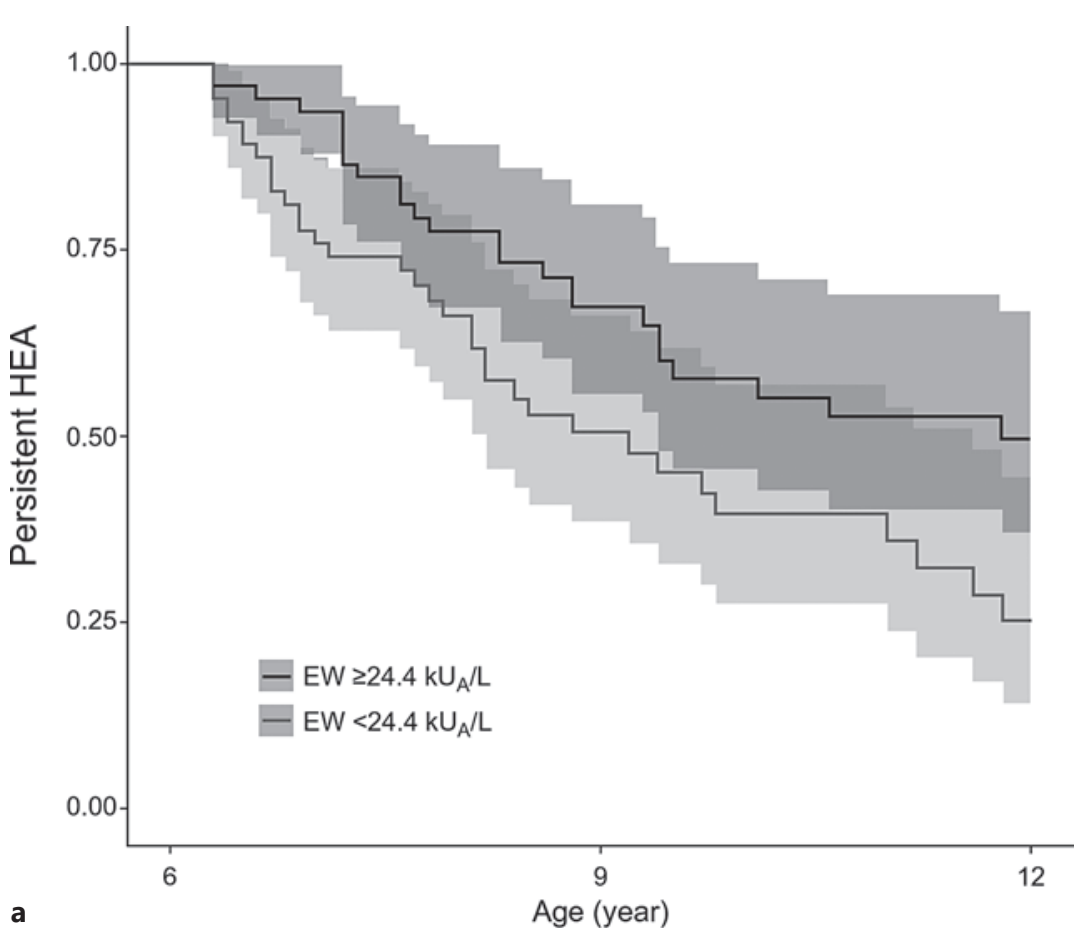

a

Age (year)

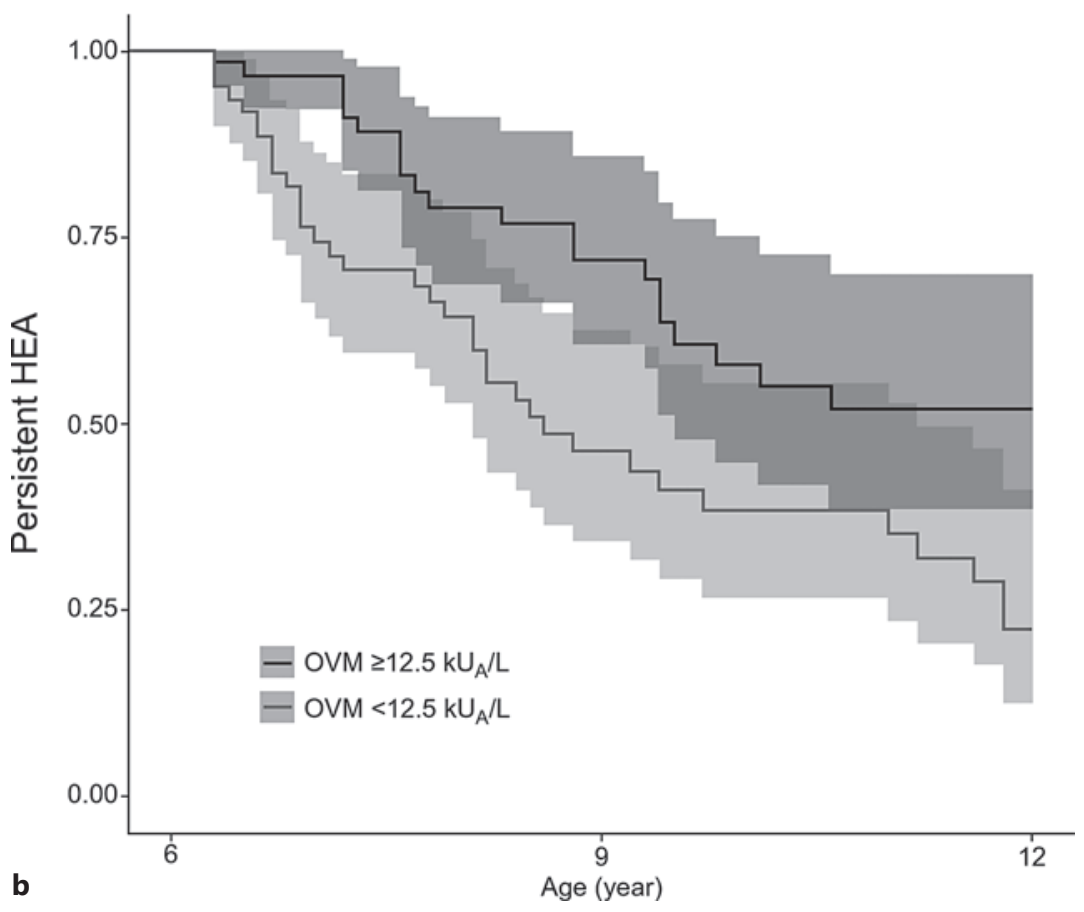

background factors of the patients in this study cannot be compared with those of patients in the previous reports, in which the factors were surveyed at a much earlier age.
Two previous studies have focused on the natural history of HEA in Japan; both were conducted at our facility $[14,15]$. Our study participants were born between 2002 and 2003, while those in the previous reports were born 
Fig. 5. Kaplan-Meyer estimation curve of the HEA resolution compared those with and without a history of anaphylaxis to $\mathrm{HE}$ (a) and those who did and did not tolerate heated egg yolk by the age of 6 years (b). a Black line indicates patients who had a history of anaphylaxis to HE. Gray line indicates patients who did not have a history of anaphylaxis to HE. b Black line indicates patients who could not tolerate heated egg yolk by the age of 6 years. Gray line indicates patients who could tolerate heated egg yolk by the age of 6 years. Shaded areas indicate 95\% CIs. HEA, hen's egg allergy; $\mathrm{HE}$, hen's egg; CIs, confidence intervals.

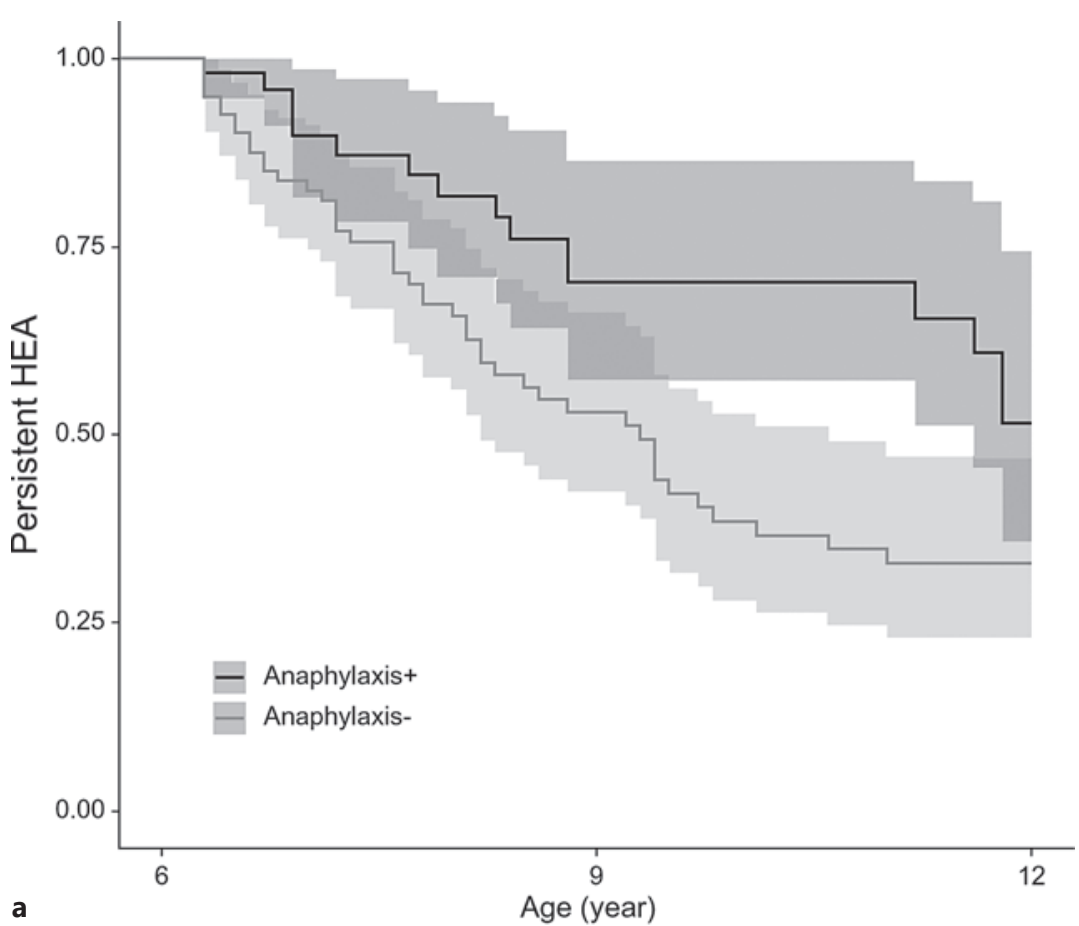

a

Age (year)

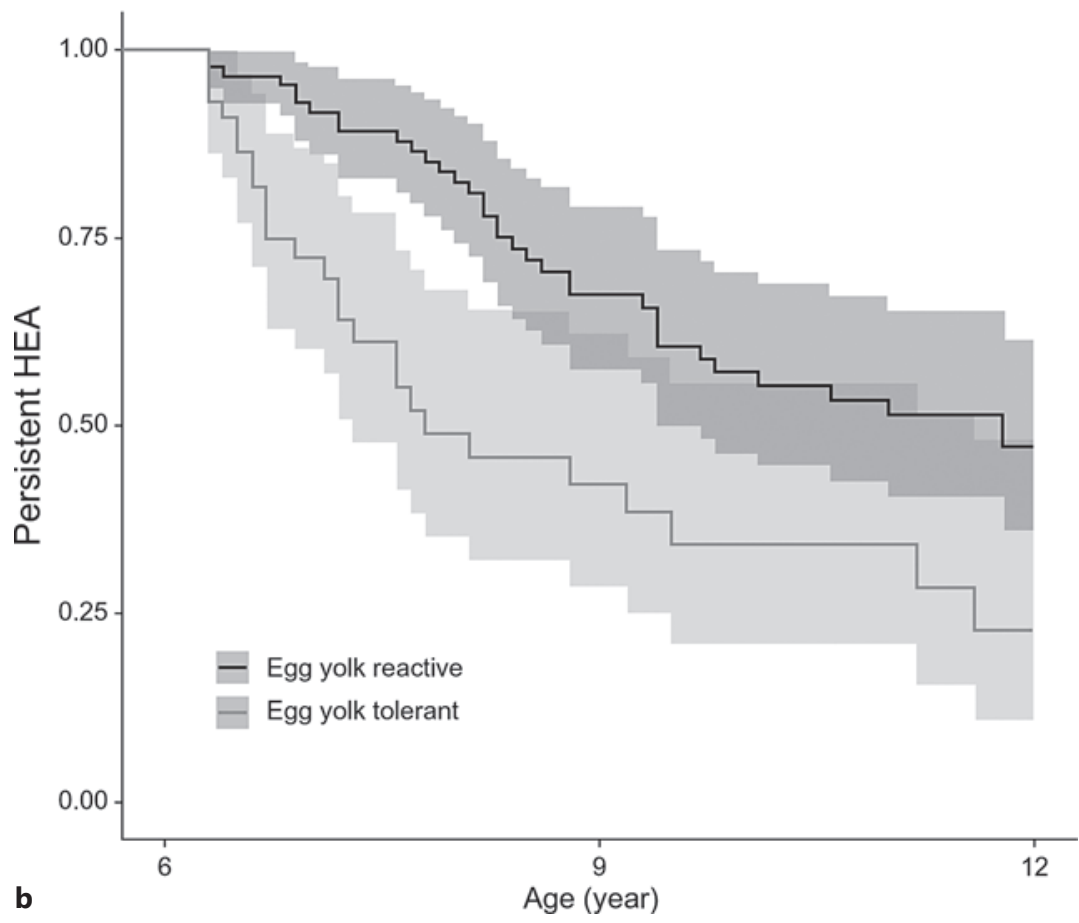

in 2005. However, our study participants also showed a similar tendency, with higher rates of comorbid bronchial asthma (62.8\%), atopic dermatitis (94.9\%), and a history of anaphylaxis to HE (40.1\%). Hence, it is possible to assume that some of the patients we followed up in this study, whose HEA persisted until 6 years of age and had a more severe form of the allergy; $60.5 \%$ of these patients acquired tolerance to HE by 12 years of age. We believe it 
is important to consider when caring for school-age patients with HEA that they still have a good chance of acquiring tolerance to $\mathrm{HE}$ beyond 6 years of age.

The use of OIT for HE was first reported in 2007 [19, 20]; since then, many studies have focused on HE OIT [21]. It has been suggested that long-term OIT may lead to sustained unresponsiveness that persists after OIT cessation [22]. We presented a Kaplan-Meier survival estimation that factored in the OIT results. This yielded better results than when those who were enrolled for OIT were treated as dropouts. This suggests that OIT may have the potential to accelerate HEA resolution.

The OIT group had significantly higher proportions of patients who experienced HE anaphylaxis and higher EW- and OVM-specific IgE values than the tolerance group. However, there were no significant differences in the patient characteristics between the OIT and persistent HEA groups. This suggests that had these patients not been enrolled for OIT, they may have had to continue with the elimination of HEA until 12 years of age. To evaluate the natural course of HEA more rigorously, we presented the Kaplan-Meier survival estimation, in which we considered patients who underwent OIT and those lost to follow-up as having "continued HE elimination until the age of 12 years" as a worst-case scenario. The rate of resolution in the real world is likely to be close to this rate.

One of the limitations of this study is its single-center design. Our facility is a tertiary center for food allergy in Japan, and patients who are referred to our facility tend to have more severe forms of food allergy than the general population. This may have affected the outcomes of this study. The other limitation is the relatively high number of patients in the OIT group and those who were lost to follow-up. This is due to the nature of this study, in which patients were followed up through standard practice; this may have resulted in dropouts due to residential address changes for familial reasons and the termination of follow-up out of the free will of the patients and their guardians. In addition, in our practice, we offered OIT as an experimental treatment option to some patients. This may be a source of bias, which might have affected the result of our study. Another limitation of this study is its design of following up patients from 6 to 12 years of age, which may make comparisons with other reports difficult. This is due to this study having been structured as a sequel to our previous study, which was focused on HEA patients aged 0-6 years [15]. Moreover, we chose 12 years of age as our end point partly because we considered the years of Japanese primary education (from 6 to 12 years of age), to be an important period when providing care for HEA children.
Although the presence of a history of HE anaphylaxis and high specific IgE values are not alterable, there may be room to intervene in the natural history of HEA by encouraging children to consume small amounts of heated HE before 6 years of age. There is a need to perform studies with a larger number of patients that focus on whether encouraging patients with HEA to consume smaller amounts of heated HE can improve the natural course of HEA.

In conclusion, $>60 \%$ of patients who still had immediate-type HEA at 6 years of age were estimated to have acquired tolerance by 12 years of age. Those who had not experienced anaphylaxis to $\mathrm{HE}$, who can tolerate heated egg yolk by the age of 6 years, and those with lower EWand OVM-specific IgE values at 6 years of age are expected to have acquired tolerance to $\mathrm{HE}$ at an earlier stage. This study provides important insights into the natural course of HEA beyond early childhood, with the acquisition of tolerance to HE continuing throughout the duration of the study. In addition, it also reveals the factors associated with persistent HEA. With the expectation that a greater number of patients would acquire tolerance to HE with age, periodical HE OFCs to confirm whether they actually acquire tolerance may be required for children with HEA beyond 6 years of age.

\section{Acknowledgements}

The authors would like to thank the current and former pediatricians, nurses, and nutritionists of the Department of Pediatrics and Clinical Research Center for Allergy and Rheumatology at Sagamihara National Hospital for their significant contributions to this study. The authors would like to thank Editage for English language editing.

\section{Statement of Ethics}

The patients or their guardians provided oral or written consent at the time of first enrollment in iAnet. Those who chose not to be enrolled were excluded from this study. The board of ethics at Sagamihara National Hospital (Rinri 2007-Oct.-009) reviewed and approved iAnet. The study was conducted in accordance with the Declaration of Helsinki.

\section{Conflict of Interest Statement}

M. Ebisawa received research support from the Health and Labor Sciences Research Grants for Research on Allergic Diseases and Immunology from the Ministry of Health, Labour, and Welfare, Japan (201414009A). M. Ebisawa also received research sup- 
port from the Practical Research Project for Allergic Disease and Immunology from the Japan Agency for Medical Research and Development, AMED (15ek0410019h0101). M. Ebisawa received lecture fees from Pfizer. M. Ebisawa is a member of the scientific advisory board of DBV Technologies. The other authors have no conflict of interest to disclose regarding this article.

\section{Funding Sources}

The work involved in the establishment of iAnet received funding from the National Hospital Organization Policy-Based Medicine Network Joint Research (Immunology) and the National Hospital Organization Network Joint Research (Immunology). The funding sources had no role in the collection and analysis of data, interpretation of the results, or decision to publish the article.

\section{Author Contributions}

H. Taniguchi performed the research, analyzed the data, and wrote the manuscript. K. Ogura supervised the data analysis and manuscript writing. N. Yanagida, S. Sato, and M. Ebisawa conceptualized the research and supervised the data analysis and manuscript writing. All the authors reviewed and approved the submitted manuscript.

\section{Data Availability Statement}

The data supporting the findings of this study are not publicly available due to their containing information that could compromise the privacy of study participants, but are available from the corresponding author, N. Yanagida, upon reasonable request.

\section{References}

1 Ebisawa M, Sugizaki C. Prevalence of pediatric allergic diseases in the first 5 years of life. J Allergy Clin Immunol. 2008 Feb;121(2 Supp 1):S237.

2 Urisu A, Ebisawa M, Ito K, Aihara Y, Ito S, Mayumi M, et al. Japanese guideline for food allergy 2014. Allergol Int. 2014 Sep;63(3): 399-419.

3 Alduraywish SA, Lodge CJ, Campbell B, Allen KJ, Erbas B, Lowe AJ, et al. The march from early life food sensitization to allergic disease: a systematic review and meta-analyses of birth cohort studies. Allergy. 2016 Jan;71(1): 77-89.

4 Eggesbø M, Botten G, Halvorsen R, Magnus $\mathrm{P}$. The prevalence of allergy to egg: a population-based study in young children. Allergy. 2001 May;56(5):403-11.

5 Peters RL, Allen KJ, Dharmage SC, Tang ML, Koplin JJ, Ponsonby AL, et al. Skin prick test responses and allergen-specific IgE levels as predictors of peanut, egg, and sesame allergy in infants. J Allergy Clin Immunol. 2013 Oct; 132(4):874-80

6 Imai T, Sugizaki C, Ebisawa M. A report on 2011 nationwide survey of immediate type food allergies in Japan (supported by a grant from "consumer affairs agency, government of Japan”). Arerugi. 2016 Jul;65(7):942-6. (Japanese).

7 Ford RP, Taylor B. Natural history of egg hypersensitivity. Arch Dis Child. 1982 Sep; 57(9):649-52.

8 Boyano-Martinez T, Garcia-Ara C, Diaz-Pena JM, Martin-Esteban M. Prediction of tolerance on the basis of quantification of egg white-specific IgE antibodies in children with egg allergy. J Allergy Clin Immunol. 2002 Aug;110(2):304-9.
9 Savage JH, Matsui EC, Skripak JM, Wood RA. The natural history of egg allergy. J Allergy Clin Immunol. 2007 Dec;120(6):1413-7.

10 Clark A, Islam S, King Y, Deighton J, Szun S, Anagnostou K, et al. A longitudinal study of resolution of allergy to well-cooked and uncooked egg. Clin Exp Allergy. 2011 May; 41(5):706-12.

11 Sicherer SH, Wood RA, Vickery BP, Jones SM, Liu AH, Fleischer DM, et al. The natural history of egg allergy in an observational cohort. J Allergy Clin Immunol. 2014 Feb; 133(2):492-9.

12 Peters RL, Dharmage SC, Gurrin LC, Koplin JJ, Ponsonby AL, Lowe AJ, et al. The natural history and clinical predictors of egg allergy in the first 2 years of life: a prospective, population-based cohort study. J Allergy Clin Immunol. 2014 Feb;133(2):485-91.

13 Xepapadaki P, Fiocchi A, Grabenhenrich L, Roberts G, Grimshaw KE, Fiandor A, et al. Incidence and natural history of hen's egg allergy in the first 2 years of life-the EuroPrevall birth cohort study. Allergy. 2016 Mar;71(3): 350-7.

14 Ikematsu K, Tachimoto H, Sugisaki C, Syukuya A, Ebisawa M. Feature of food allergy developed during infancy (2)-acquisition of tolerance against hen's egg, cow's milk, wheat, and soybean up to 3 years old. Arerugi. 2006 May;55(5):533-41. (Japanese).

15 Ohtani K, Sato S, Syukuya A, Asaumi T, Ogura K, Koike Y, et al. Natural history of immediate-type hen's egg allergy in Japanese children. Allergol Int. 2016 Apr;65(2):153-7.
16 Okada Y, Yanagida N, Sato S, Ebisawa M. Heated egg yolk challenge predicts the natural course of hen's egg allergy: a retrospective study. World Allergy Organ J. 2016 Oct 6; 9(1):31.

17 Yanagida N, Sato S, Asaumi T, Ogura K, Borres MP, Ebisawa M. Safety and feasibility of heated egg yolk challenge for children with egg allergies. Pediatr Allergy Immunol. 2017 Jun;28(4):348-54.

18 Leonard SA, Sampson HA, Sicherer SH, Noone S, Moshier EL, Godbold J, et al. Dietary baked egg accelerates resolution of egg allergy in children. J Allergy Clin Immunol. 2012 Aug;130(2):473-80.e1.

19 Buchanan AD, Green TD, Jones SM, Scurlock AM, Christie L, Althage KA, et al. Egg oral immunotherapy in nonanaphylactic children with egg allergy. J Allergy Clin Immunol. 2007 Jan;119(1):199-205.

20 Morisset M, Moneret-Vautrin DA, Guenard L, Cuny JM, Frentz P, Hatahet R, et al. Oral desensitization in children with milk and egg allergies obtains recovery in a significant proportion of cases. A randomized study in 60 children with cow's milk allergy and 90 children with egg allergy. Eur Ann Allergy Clin Immunol. 2007 Jan;39(1):12-9.

21 Wood RA. Food allergen immunotherapy: current status and prospects for the future. Allergy Clin Immunol. 2016 Apr;137(4):97382.

22 Jones SM, Burks AW, Keet C, Vickery BP, Scurlock AM, Wood RA, et al. Long-term treatment with egg oral immunotherapy enhances sustained unresponsiveness that persists after cessation of therapy. J Allergy Clin Immunol. 2016 Apr;137(4):1117-27.e10. 